Introduction: Triple-negative breast cancer (TNBC) is a markedly aggressive molecular subtype of breast cancer; there is an urgent need to clarify the molecular mechanisms underlying the progression and metastases of $B L B C$, in order to find a novel targeted therapy. Microfibrillar-associated protein 5 (MFAP5) plays an essential role in the regulation of cell behaviour and survival. Integral membrane protein 2A (ITM2A) is a type II transmembrane protein, which is a member of a family of autophagy related proteins.

The aim of this study was to assess the expression of MFAP5 and ITM2A proteins in tissues of BLBC using immunohistochemistry, in order to correlate the expression with clinicopathological and prognostic parameters of such aggressive cancer.

Material and methods: The present study included sections from archived paraffin blocks retrieved from 120 patients with TNBC. We collected cases from three years, i.e. from 2016 to 2019. We assessed expression of MFAP5 and ITM2A using immunohistochemistry.

Results: High expression of MFAP5 and low expression of ITM2A was associated with advanced stage $(p=0.007)$, higher grade of tumour $(p=0.005$ and $p=0.004$, respectively), the presence of lymph nodes metastases $(p<0.001$ and $p=0.002$, respectively), lower three-year RFS rate $(p<0.001$ and $p=0.016$, respectively), and lower three-year OS rate $(p<0.001)$.

Conclusions: MFAP5 and ITM2A are novel prognostic biomarkers for breast cancer and might be considered as promising therapeutic targets for patients with breast cancer, particularly TNBC molecular subtype, in the future.

Key words: MFAP5, ITM2A, triple-negative breast cancer, prognosis, immunohistochemistry.

Contemp Oncol (Pozn) 2020; 24 (2): 87-95 DOI: https://doi.org/10.5114/wo.2020.97520

\section{Prognostic and clinicopathological values of tissue expression of MFAP5 and ITM2A in triple-negative breast cancer: an immunohistochemical study}

\author{
Abdulwahab A. Abuderman'1, Ola A. Harb², Loay M. Gertallah ${ }^{3}$ \\ ${ }^{1}$ Department of Basic Medical Sciences, College of Medicine, Prince Sattam \\ Bin Abdulaziz University, Al Kharj, Saudi Arabia \\ ${ }^{2}$ Department of Pathology, Faculty of Medicine, Zagazig University, Zagazig, Egypt \\ ${ }^{3}$ Department of General Surgery, Faculty of Medicine, Zagazig University, Zagazig, Egypt
}

\section{Introduction}

Triple-negative breast cancer (TNBC) is an aggressive molecular subtype of breast cancer and is characterised by negative oestrogen receptor (OR), progesterone receptor (PR), and HER2-neu [1]. It has an aggressive behaviour and unfavourable prognostic criteria such as high grade, early brain and lung metastasis, dismal outcome, and unfavourable survival rates [2]. There is still no specific targeted therapy for TNBC, and it is even chemo-resistant [3]. Hence, there is an urgent need to clarify the molecular mechanisms underlying the progression and metastases of BLBC, in order to detect novel targeted therapies. The promoting factors of the epithelial-mesenchymal transition (EMT) process were found to be negative prognostic factors in cancer of the breast [4]. There are many factors that control the EMT process and cancer progression. There is a plethora of signalling pathways that are incriminated in the activation of EMT [5]. Microfibrillar-associated protein 5 (MFAP5) is a protein with multiple functions, which plays an essential role in the regulation of cell behaviour and survival [1]. MFAP5 is one of the expressed genes in most human malignancies; a few studies have clarified its function [6]. Evaluation of MFAP5 expression in malignant epithelial cells is detailed in only a few studies [7]. Autophagy is a stress-related process that degrades dangerous cytosolic components, and it is characterised by phagophore formation. It was found that factors enhancing autophagy could control cancer progression [8]. Integral membrane protein 2A (ITM2A) is a type II transmembrane protein, which is one of the autophagy related proteins, and its role in breast cancer, particularly TNBC, remains uncertain [9].

The aim of the present study was to assess the expression of MFAP5 and ITM2A proteins in tissues of TNBC using immunohistochemistry, to correlate the expression with clinicopathological and prognostic parameters of such an aggressive cancer, and to show the relationship between EMT and autophagy in breast cancer progression and metastasis.

\section{Material and methods}

The present study is a retrospective study that included sections from archived paraffin blocks retrieved from 120 patients with TNBC. We collected cases during three years, from December 2016 to December 2019, from Zagazig University hospitals and other private surgical hospitals. We obtained approval from the local Ethics Committee of ZU institutional board for performance of the study. Patients signed a written informed consent 
form to be included in the study. For all patients ER, PR, Her2-neu, and Ki-67 labelling index were assessed. We recorded all clinicopathological and follow-up data from patients' files. Staging of samples derived from included cases was done using the $7^{\text {th }}$ edition of the TNM staging system [10]. Grading of cases was done according to the grading criteria of Elston and Ellis [11].

For immunohistochemistry staining we used primary rabbit poly clonal anti-MFAP5 antibody (catalogue number; MBS9204084) and primary rabbit poly clonal anti-ITM2A antibody (catalogue number; ab203620) at $1: 50$ dilution.

Inclusion criteria: Samples derived from patients with TNBC who showed negative expression of ER, PR, and Her2neu and have full histopathological and follow-up data.

Exclusion criteria: Paraffin blocks with insufficient samples, patients with incomplete clinicopathological or follow-up data, and patients with positive ER, PR, and Her2neu expression.

We assessed nuclear expression of MFAP5 and cytoplasmic expression of ITM2A in malignant epithelial cells semiquantitively, giving the stain intensity scores: 0,1 , 2 , and 3 - representing: negative, weak, moderate, and strong stain, respectively, and giving the stain extent in the tumour cells scores: $0,1,2,3$, and 4 - representing: $0 \%$, from 1 to $25 \%$, from $26-50 \%$, from $51-75 \%$, and more than $75 \%$, respectively [9].

We multiplied scores of intensity and extent to reach the final stain score from $0-12$, then finally the stain was divided into low and high according to the cut-off point of 4; this cut-off point value was used to divide results into low and high results to allow easy and clear statistical analysis of values.

We used sections from skeletal muscles and normal colon mucosa as positive controls for MFAP5 and ITM2A, respectively.

Patients clinicopathological data, such as age and sex of patients, tumour size, grade, stage, and the presence of lymph node metastases and distant metastases, and follow-up data such as disease progression, recurrence, response to chemotherapy, and survival rates, were retrospectively collected, recorded, tabulated, and statistically analysed to correlate between MFAP5 and ITM2A expression and the data.

\section{Statistical analysis}

We performed statistical analysis using SPSS 22.0 (SPSS Inc., Chicago, IL, USA).

We determined continuous variables as mean $\pm S D$, and we have checked them for normality by using the Shapiro-Wilk test. We determined categorical variables as numbers and percentages. We used the Mann-Whitney $U$ test to allow easy comparison between non-normally distributed variables, while the $\chi^{2}$ test was used to compare trends of changes in frequencies of ordinal data. Stratification and correlation of rates of OS and RFS rates with the immunohistochemical markers were made by Kaplan-Meier curve analysis.

\section{Results}

MFAP5 expression and its relationship with pathological findings which were found in samples retrieved from included cases are shown in Table 1 and Figure 1. MFAP5 tissue expression in TNBC cells was positively correlated with age of patients, presence of lymph node metastases $(p<0.001)$, larger tumour size $(p=0.004)$, and higher stage $(p=0.007)$ and higher grade of tumour $(p=0.005)$.

ITM2A expression and its relationship with pathological findings which were found in samples retrieved from included cases are shown in Table 2 and Figure 2. Because we did not correlate malignant tissue with non-neoplastic breast tissue, down-regulation of ITM2A expression in TNBC was found - its expression was decreased in higher grade than in lower grade tumour and areas of intra-ductal carcinoma, reaching negative results in high-grade tumours. ITM2A tissue expression in TNBC cells was negatively correlated with the age of patients $(p=0.009)$, presence of lymph node metastases $(p=0.002)$, larger tumour size $(p=0.006)$, and higher stage $(p=0.007)$ and higher grade of tumour $(p=0.004)$.

We found an inverse relationship between expression of both MFAP5 and ITM2A in TNBC tumour cells $(r=-0.640 ; p=0.002)$ (Fig. 3).

Patient outcomes, follow-up, and survival data results are shown in Table 3 and 4 and in Figure 4 and 5.

Recurrence-free survival rate (RFS) and overall survival rate (OS) for all patients were $83.6 \%$ and $60.8 \%$, respectively. We assessed the recurrence of the disease and the RFS rate only in patients who had complete response to treatment.

In patients underwent chemotherapy, low MFAP5 expression and high ITIM2A expression was positively associated with good response to chemotherapy. High MFAP5 expression and low ITM2A expression was associated with recurrence of tumour and disease progression ( $p=0.002$ and 0.007, respectively). High MFAP5 expression and low ITM2A expression was associated with a low three-year RFS rate ( $p=0.001$ and 0.016 , respectively). High MFAP5 expression and low ITM2A expression was associated with a low three-year OS rate $(p<0.001)$.

\section{Discussion}

TNBC, which is a molecular subtype of breast cancer, has aggressive behaviour, no targeted therapy, and high incidence of disease progression and development of metastases; hence, it needs further evaluation and new studies to detect novel prognostic markers and targeted therapy to improve its outcome [1]. EMT initiation and activation in tumour cells is thought to be responsible for starting and maintaining the metastatic program [12]. Studying biomarkers that play a role in EMT might lead to the detection of novel biomarkers that could help to improve the prognosis of TNBC.

MFAP5 gene expression was previously found to be associated with poor outcome of many tumours through activation of signalling pathways that are responsible for EMT induction and maintenance in tumour cells, but its tissue protein expression by immunohistochemistry in epithelial cells is not fully detailed. 
Table 1. Association between clinicopathological findings and MFAP5 in included triple-negative breast cancer patients

\begin{tabular}{|c|c|c|c|c|c|c|c|}
\hline \multirow[t]{3}{*}{ Characteristics } & \multirow{3}{*}{$\begin{array}{l}\text { All }(n=120) \\
\quad n(\%)\end{array}$} & \multicolumn{2}{|c|}{ MFAP5 } & \multirow[t]{3}{*}{$p$-value } & \multicolumn{2}{|c|}{ MFAP5 (\%) } & \multirow[t]{3}{*}{$p$-value } \\
\hline & & $\begin{array}{c}\text { Low } \\
(n=46)\end{array}$ & $\begin{array}{c}\text { High } \\
(n=74)\end{array}$ & & \multirow[t]{2}{*}{ Mean $\pm S D$} & \multirow[t]{2}{*}{ Median (range) } & \\
\hline & & $n(\%)$ & $n(\%)$ & & & & \\
\hline \multicolumn{8}{|l|}{ Age (years) } \\
\hline Mean \pm SD & $56.23 \pm 10.99$ & $49.91 \pm 8.72$ & $60.16 \pm 10.50$ & \multirow[t]{2}{*}{$<0.001^{a}$} & & & \\
\hline Median (range) & $56(39-87)$ & $45(39-67)$ & $60(40-87)$ & & & & \\
\hline$\leq 50$ years & $46(38.3)$ & $30(65.2)$ & $16(21.6)$ & \multirow[t]{2}{*}{$0.001^{b}$} & $20.21 \pm 20.62$ & $12(0-56)$ & \multirow[t]{2}{*}{$<0.001^{a}$} \\
\hline$>50$ years & $74(61.7)$ & $16(34.8)$ & $58(78.4)$ & & $62.97 \pm 28.18$ & 75 (0-97) & \\
\hline \multicolumn{8}{|l|}{ Size $(\mathrm{cm})$} \\
\hline Mean \pm SD & $6.33 \pm 3.59$ & $4.04 \pm 2.71$ & $7.75 \pm 3.34$ & \multirow[t]{2}{*}{$<0.001^{a}$} & & & \\
\hline Median (range) & $7(1-13)$ & $4(1-9)$ & $8(1.5-13)$ & & & & \\
\hline$<5 \mathrm{~cm}$ & $48(40)$ & $30(65.2)$ & $18(24.3)$ & \multirow[t]{2}{*}{$0.004^{b}$} & $20.79 \pm 20.37$ & $12(0-56)$ & \multirow[t]{2}{*}{$<0.001^{a}$} \\
\hline$\geq 5 \mathrm{~cm}$ & $72(60)$ & $16(34.8)$ & $56(75.7)$ & & $63.77 \pm 28.15$ & 76.50 (0-97) & \\
\hline \multicolumn{8}{|l|}{ Grade } \\
\hline Grade I & $20(16.7)$ & $14(30.4)$ & $6(8.1)$ & \multirow[t]{3}{*}{$0.005^{b}$} & $15.20 \pm 20.61$ & $0(0-45)$ & \multirow[t]{3}{*}{$<0.001^{a}$} \\
\hline Grade II & $30(25)$ & 18 (39.1) & $12(16.2)$ & & $24.46 \pm 19.28$ & $20(0-56)$ & \\
\hline Grade III & $70(58.3)$ & $14(30.4)$ & $56(75.7)$ & & $65.02 \pm 27.53$ & $78(0-97)$ & \\
\hline \multicolumn{8}{|l|}{ Lymph node } \\
\hline Negative & 38 (31.7) & $38(82.6)$ & $0(0)$ & \multirow[t]{2}{*}{$<0.001^{b}$} & $9.52 \pm 9.23$ & $10(0-22)$ & \multirow[t]{2}{*}{$<0.001^{\mathrm{a}}$} \\
\hline Positive & $82(68.3)$ & $8(17.4)$ & 74 (100) & & $63.75 \pm 24.58$ & $60(0-97)$ & \\
\hline \multicolumn{8}{|l|}{$T$} \\
\hline $\mathrm{T} 1$ & $26(21.7)$ & 18 (39.1) & $8(10.8)$ & \multirow[t]{4}{*}{$0.003^{b}$} & $16.84 \pm 18.20$ & $11(0-50)$ & \multirow[t]{4}{*}{$<0.001^{a}$} \\
\hline T2 & $22(18.3)$ & $12(26.1)$ & $10(13.5)$ & & $25.45 \pm 22.64$ & $22(0-56)$ & \\
\hline T3 & $44(36.7)$ & $16(34.8)$ & $28(37.8)$ & & $49.54 \pm 27.38$ & $52(0-95)$ & \\
\hline T4 & $28(23.3)$ & $0(0)$ & $28(37.8)$ & & $86.14 \pm 6.45$ & 87 (75-97) & \\
\hline N & & & & & & & \\
\hline NO & 38 (31.7) & $38(82.6)$ & $0(0)$ & $0.003^{b}$ & $9.52 \pm 9.23$ & $10(0-22)$ & $<0.001^{\mathrm{a}}$ \\
\hline N1 & $14(11.7)$ & $4(8.7)$ & $10(13.5)$ & & $34 \pm 18.43$ & $34(0-50)$ & \\
\hline N2 & $42(35)$ & $2(4.3)$ & $40(54.1)$ & & $64.33 \pm 20.86$ & 60 (24-95) & \\
\hline N3 & $26(21.7)$ & $2(4.3)$ & $24(32.4)$ & & $78.84 \pm 18.84$ & 80 (24-97) & \\
\hline Stage & & & & & & & \\
\hline Stage I & $18(15)$ & 18 (39.1) & $0(0)$ & $0.007^{b}$ & $6.11 \pm 5.84$ & $10(0-12)$ & $<0.001^{\mathrm{a}}$ \\
\hline Stage II & $28(23.3)$ & $20(43.5)$ & $8(10.8)$ & & $20.71 \pm 16.87$ & $21(0-50)$ & \\
\hline Stage III & $42(35)$ & $8(17.4)$ & $34(45.6)$ & & $49.95 \pm 20.15$ & $50(0-80)$ & \\
\hline Stage IV & $32(26.7)$ & $0(0)$ & $32(43.2)$ & & $87.56 \pm 6.21$ & $89.50(80-97)$ & \\
\hline ITM2A & & & & & & & \\
\hline Mean \pm SD & $40 \pm 33.94$ & $68.26 \pm 25.16$ & $22.43 \pm 25.96$ & $<0.001^{\mathrm{a}}$ & & & \\
\hline Median (range) & $25(0-90)$ & $80(0-90)$ & $20(0-90)$ & & & & \\
\hline Low & $64(53.3)$ & $0(0)$ & $64(86.5)$ & $<0.001^{b}$ & $72.75 \pm 18.13$ & $80(40-97)$ & $<0.001^{\mathrm{a}}$ \\
\hline High & $56(46.7)$ & $46(100)$ & 10 (13.5) & & $16.67 \pm 15.81$ & $16(0-50)$ & \\
\hline
\end{tabular}

${ }^{a}$ Mann-Whitney $U$ test, ${ }^{b} \chi^{2}$ test

We found that MFAP5 tissue expression in TNBC cells was associated with unfavourable pathological data and dismal outcome, similarly to previous studies [1, 13-15].

Wu et al. [1] showed that MFAP5 played essential roles in TNBC occurrence, invasion, and progression and that its up-regulation is associated with advanced TNM staging and higher incidence of metastases to axillary lymph nodes. Moreover, Wu et al. [15] revealed that MFAP5 mRNA levels were markedly up-regulated.

There are many studies showing that MFAP5 up-regulation is related to progression of many tumours and poor prognosis [13-16]. Our study might help in clarifying the mechanisms by which overexpression of MFAP5 in TNBC leads to its progression. 

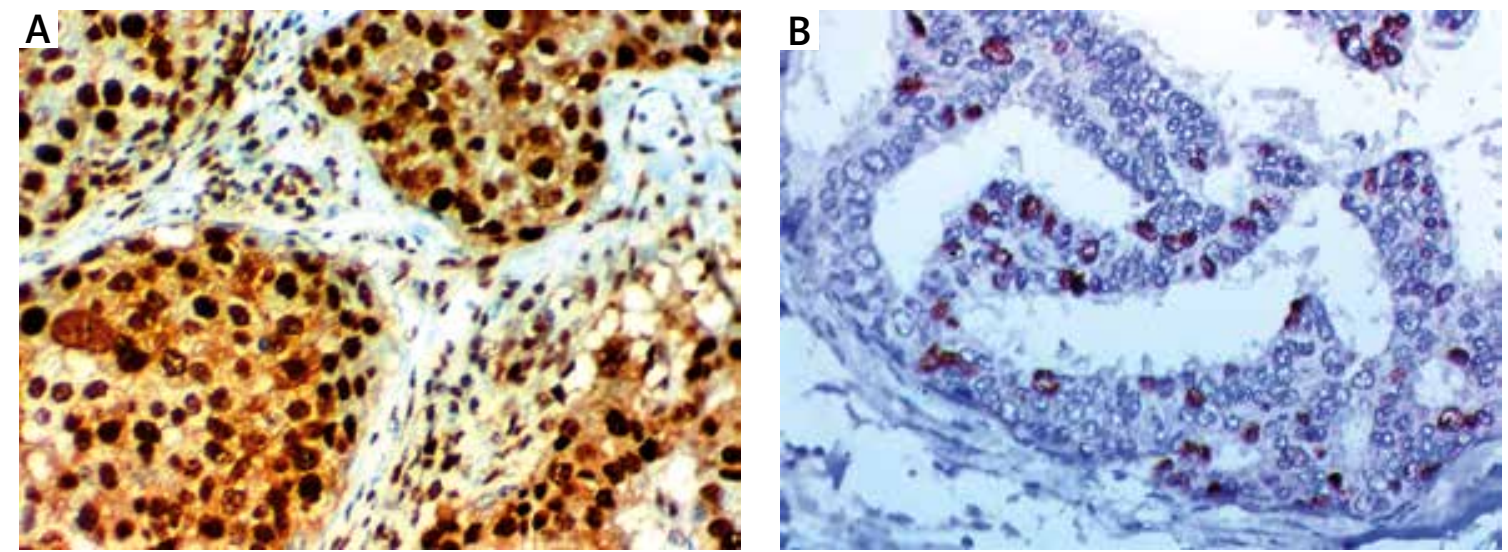

Fig. 1. Nuclear expression of MFAP5: (A) high expression in cells of triple-negative breast cancer (TNBC); high grade, stage IV $\times 400$, (B) low nuclear expression in intra-ductal carcinoma $\times 400$

Table 2. Association between clinicopathological findings and ITM2A in included triple-negative breast cancer patients

\begin{tabular}{|c|c|c|c|c|c|c|c|}
\hline \multirow[t]{3}{*}{ Characteristics } & \multirow[t]{2}{*}{ All $(n=120)$} & \multicolumn{2}{|c|}{ ITM2A } & \multirow[t]{3}{*}{$p$-value } & \multicolumn{2}{|c|}{ ITM2A (\%) } & \multirow[t]{3}{*}{$p$-value } \\
\hline & & Low $(n=64)$ & High $(n=56)$ & & \multirow[t]{2}{*}{ Mean \pm SD } & \multirow[t]{2}{*}{ Median (range) } & \\
\hline & $n(\%)$ & $n(\%)$ & $n(\%)$ & & & & \\
\hline \multicolumn{8}{|l|}{ Age (years) } \\
\hline Mean $\pm S D$ & $56.23 \pm 10.99$ & $61.28 \pm 10.77$ & $50.46 \pm 8.14$ & \multirow[t]{2}{*}{$<0.001^{a}$} & & & \\
\hline Median (range) & $56(39-87)$ & $60(40-87)$ & 49 (39-67) & & & & \\
\hline$\leq 50$ years & $46(38.3)$ & $10(15.6)$ & $18(64.3)$ & \multirow[t]{2}{*}{$0.009^{b}$} & $67.82 \pm 24.57$ & $80(20-90)$ & \multirow[t]{2}{*}{$<0.001^{a}$} \\
\hline$>50$ years & $74(61.7)$ & $54(84.4)$ & $10(35.7)$ & & $22.70 \pm 26.77$ & $15(0-90)$ & \\
\hline \multicolumn{8}{|l|}{ Size $(\mathrm{cm})$} \\
\hline Mean \pm SD & $6.33 \pm 3.59$ & $8.50 \pm 2.70$ & $3.85 \pm 2.82$ & \multirow[t]{2}{*}{$<0.001^{a}$} & & & \\
\hline Median (range) & $7(1-13)$ & $9(3-13)$ & $3(1-9)$ & & & & \\
\hline$<5 \mathrm{~cm}$ & $48(40)$ & $10(15.6)$ & $19(67.9)$ & \multirow[t]{2}{*}{$0.006^{b}$} & $68.75 \pm 24.46$ & 80 (20-90) & \multirow[t]{2}{*}{$<0.001^{a}$} \\
\hline$\geq 5 \mathrm{~cm}$ & $72(60)$ & $54(84.4)$ & $9(32.1)$ & & $20.83 \pm 24.58$ & $15(0-80)$ & \\
\hline \multicolumn{8}{|l|}{ Grade } \\
\hline Grade I & $20(16.7)$ & $6(9.4)$ & $7(25)$ & \multirow[t]{3}{*}{$0.004^{b}$} & $59 \pm 25.47$ & $70(20-80)$ & \multirow[t]{3}{*}{$<0.001^{a}$} \\
\hline Grade II & $30(25)$ & $4(6.3)$ & $13(46.4)$ & & $76 \pm 21.23$ & $80(25-90)$ & \\
\hline Grade III & $70(58.3)$ & $54(84.4)$ & $8(28.6)$ & & $19.14 \pm 22.72$ & $15(0-80)$ & \\
\hline \multicolumn{8}{|l|}{ Lymph node } \\
\hline Negative & 38 (31.7) & $0(0)$ & $19(67.9)$ & \multirow[t]{2}{*}{$0.002^{b}$} & $78.42 \pm 7.64$ & $80(60-90)$ & \multirow[t]{2}{*}{$<0.001^{a}$} \\
\hline Positive & $82(68.3)$ & $64(100)$ & $9(32.1)$ & & $22.19 \pm 25.43$ & $20(0-90)$ & \\
\hline \multicolumn{8}{|l|}{$\mathrm{T}$} \\
\hline $\mathrm{T} 1$ & $26(21.7)$ & $0(0)$ & $13(46.4)$ & \multirow[t]{4}{*}{$<0.001^{\mathrm{b}}$} & $82.30 \pm 4.38$ & 80 (80-90) & \multirow[t]{4}{*}{$<0.001^{\circ}$} \\
\hline $\mathrm{T} 2$ & $22(18.3)$ & $10(15.6)$ & $6(21.4)$ & & $52.72 \pm 28.84$ & $60(20-90)$ & \\
\hline T3 & $44(36.7)$ & $26(40.6)$ & $9(32.1)$ & & $29.95 \pm 27.21$ & $23(0-80)$ & \\
\hline $\mathrm{T} 4$ & $28(23.3)$ & $28(43.8)$ & $0(0)$ & & $6.50 \pm 8.33$ & $0(0-24)$ & \\
\hline \multicolumn{8}{|l|}{$N$} \\
\hline NO & 38 (31.7) & $0(0)$ & 19 (67.9) & \multirow[t]{4}{*}{$0.005^{b}$} & $78.42 \pm 7.64$ & $80(60-90)$ & \multirow[t]{4}{*}{$<0.001^{a}$} \\
\hline N1 & 14 (11.7) & $0(0)$ & $7(25)$ & & $70 \pm 21.60$ & $80(40-90)$ & \\
\hline N2 & $42(35)$ & $40(62.5)$ & $1(3.6)$ & & $16.14 \pm 10.18$ & $20(0-25)$ & \\
\hline N3 & $26(21.7)$ & $24(37.5)$ & $1(3.6)$ & & $6.23 \pm 8.81$ & $0(0-22)$ & \\
\hline Stage & & & & & & & \\
\hline Stage I & 18 (15) & $0(0)$ & $9(32.1)$ & $0.007^{b}$ & $81.11 \pm 3.33$ & $80(80-90)$ & $<0.001^{\mathrm{a}}$ \\
\hline Stage II & $28(23.3)$ & $0(0)$ & $14(50)$ & & $78.57 \pm 9.49$ & 80 (60-90) & \\
\hline Stage III & $42(35)$ & $32(50)$ & $5(17.9)$ & & $21.85 \pm 16.49$ & $24(0-70)$ & \\
\hline Stage IV & $32(26.7)$ & $32(50)$ & $0(0)$ & & $6.93 \pm 7.46$ & $5(0-20)$ & \\
\hline
\end{tabular}



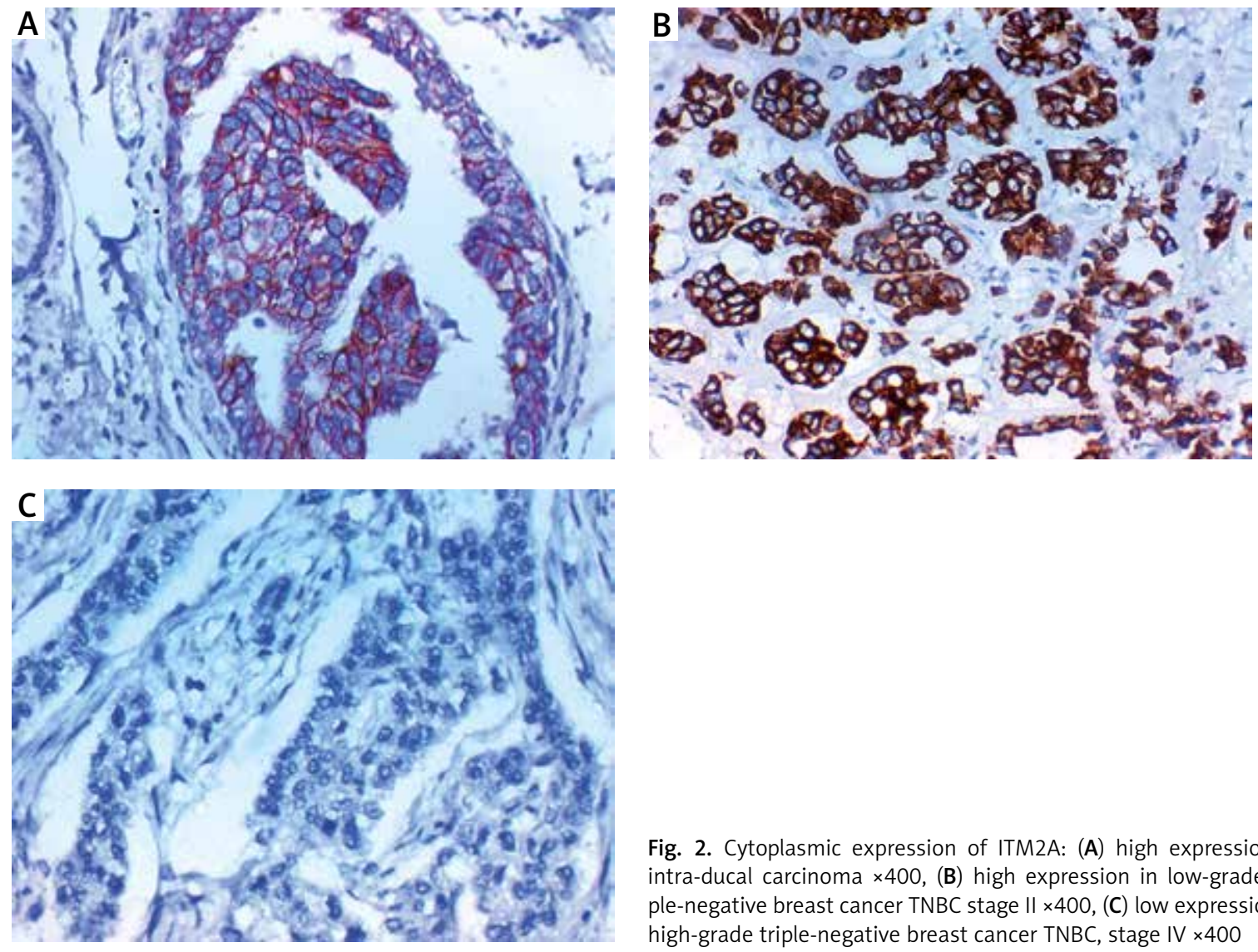

Fig. 2. Cytoplasmic expression of ITM2A: (A) high expression in intra-ducal carcinoma $\times 400$, (B) high expression in low-grade triple-negative breast cancer TNBC stage II $\times 400$, (C) low expression in high-grade triple-negative breast cancer TNBC, stage IV $\times 400$

MFAP5 leads to activation of Notch and TGF- $\beta$ signalling pathways, which lead to induction and promotion of EMT, which in turn lead to an aggressive course and progression of tumour cells to invasion and metastasis [1].

Our results confirm previous results about expression of MFAP5 [17] and activation of Notch pathway, which means that targets against such a gene may lead to blocking the Notch pathway and stopping tumour progression, which might improve the prognosis of BLBC.

MFAP5 leads to tumour progression by increasing expression of mesenchymal markers, such as N-cadherin, and by decreasing E-cadherin levels in cells of TNBC that promote EMT. This denotes that MFAP5 induces EMT in TNBC cells. Additionally, MFAP5 promoted activation of Smad and the Notch signalling pathway downstream signals, and that inhibition of Notch signalling pathway through down-regulation of MFAP5 leads to inhibition of EMT and tumour progression [1].

It was found that inhibitors of Notch and TGF- $\beta$ signalling pathways leads to blocking of MFAP5-mediated invasion, migration, and proliferation of tumour cells.

We proved the results of Wu et al. [1] regarding the association between MFAP5 and EMT activation, but all the mechanisms of action of MFAP5 remain unknown. Hence, we assessed the expression of another marker, i.e. ITM2A, which was found to be associated with autophagy induction because we hypothesised that there could be a relationship between MFAP5 expression and autophagy control in TNBC tumour cells.

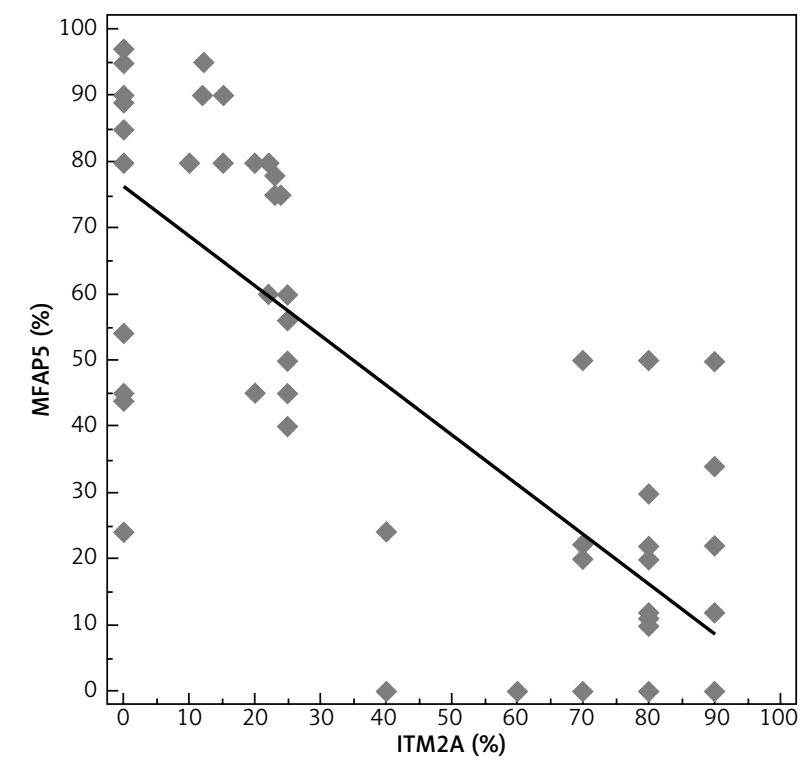

Fig. 3. Scatter plot with regression line shows a significant strong inverse correlation between MFAP5 and ITM2A

ITM2A was recently described to have cancer suppressor function in many epithelial cancers [18], but its role in autophagy and cancer progression has not been established in TNBC. In the current study we found that the expression of ITM2A is markedly reduced in malignant tissue, and its expression is associated with favourable parameters. Moreover, ITM2A was inversely associated with 
Table 3. Association between clinicopathological features, MFAP5, ITM2A, and survival in included triple-negative breast cancer patients

\begin{tabular}{|c|c|c|c|c|c|c|c|}
\hline \multirow[t]{2}{*}{ Characteristics } & \multirow{2}{*}{$\begin{array}{c}\text { All } \\
(n=120)\end{array}$} & \multicolumn{2}{|c|}{ Recurrence-free survival } & \multirow[t]{2}{*}{$p$-value } & \multicolumn{2}{|c|}{ Overall survival } & \multirow[t]{2}{*}{$p$-value } \\
\hline & & $\begin{array}{l}\text { Median RFS } \\
\text { (months) }\end{array}$ & $\begin{array}{c}\text { 3-year RFS } \\
(\%)\end{array}$ & & $\begin{array}{c}\text { Median OS } \\
\text { (months) }\end{array}$ & 3-year OS (\%) & \\
\hline All patients & $n(\%)$ & NR & 83.6 & & NR & 60.8 & \\
\hline \multicolumn{8}{|l|}{ Age (years) } \\
\hline$\leq 50$ years & $46(38.3)$ & NR & 91.3 & \multirow[t]{2}{*}{$0.119^{*}$} & NR & 91.3 & \multirow[t]{2}{*}{$<0.001^{*}$} \\
\hline$>50$ years & 74 (61.7) & NR & 75 & & 24 & 41.1 & \\
\hline \multicolumn{8}{|l|}{ Size $(\mathrm{cm})$} \\
\hline$<5 \mathrm{~cm}$ & $48(40)$ & NR & 91.7 & \multirow[t]{2}{*}{$0.087^{*}$} & NR & 91.7 & \multirow[t]{2}{*}{$<0.001^{*}$} \\
\hline$\geq 5 \mathrm{~cm}$ & $72(60)$ & NR & 73.7 & & 24 & 39.4 & \\
\hline \multicolumn{8}{|l|}{ Grade } \\
\hline Grade I & $20(16.7)$ & NR & 100 & \multirow[t]{3}{*}{$0.139^{*}$} & NR & 100 & \multirow[t]{3}{*}{$<0.001^{\star}$} \\
\hline Grade II & $30(25)$ & NR & 86.7 & & NR & 86.7 & \\
\hline Grade III & $70(58.3)$ & NR & 72.2 & & 24 & 37.6 & \\
\hline \multicolumn{8}{|l|}{ Lymph node } \\
\hline Negative & $38(31.7)$ & NR & 100 & \multirow[t]{2}{*}{$0.010^{*}$} & NR & 100 & \multirow[t]{2}{*}{$<0.001^{*}$} \\
\hline Positive & $82(68.3)$ & NR & 70.4 & & 27 & 41.6 & \\
\hline \multicolumn{8}{|l|}{$\mathrm{T}$} \\
\hline $\mathrm{T} 1$ & $26(21.7)$ & NR & 84.6 & \multirow[t]{4}{*}{$0.028^{*}$} & $N R$ & 84.6 & \multirow[t]{4}{*}{$<0.001^{*}$} \\
\hline $\mathrm{T} 2$ & $22(18.3)$ & NR & 100 & & NR & 100 & \\
\hline T3 & $44(36.7)$ & NR & 77.8 & & NR & 62 & \\
\hline T4 & $28(23.3)$ & 20 & 0 & & 17 & 0 & \\
\hline \multicolumn{8}{|l|}{$N$} \\
\hline NO & 38 (31.7) & $N R$ & 100 & \multirow[t]{4}{*}{$0.006^{*}$} & $N R$ & 100 & \multirow[t]{4}{*}{$<0.001^{*}$} \\
\hline N1 & $14(11.7)$ & $N R$ & 71.4 & & $N R$ & 71.4 & \\
\hline N2 & $42(35)$ & NR & 78.6 & & 28 & 45.4 & \\
\hline N3 & $26(21.7)$ & 20 & 33.3 & & 17 & 20.5 & \\
\hline \multicolumn{8}{|l|}{ Stage } \\
\hline Stage I & $18(15)$ & NR & 100 & \multirow[t]{4}{*}{$0.226^{*}$} & NR & 100 & \multirow[t]{4}{*}{$<0.001^{*}$} \\
\hline Stage II & $28(23.3)$ & NR & 85.7 & & NR & 85.7 & \\
\hline Stage III & $24(35)$ & NR & 75 & & NR & 66.7 & \\
\hline Stage IV & $32(26.7)$ & - & - & & 17.5 & 0 & \\
\hline MFAP5 & & & & & & & \\
\hline Low & $46(38.3)$ & $N R$ & 100 & $0.001^{\star}$ & NR & 100 & $<0.001^{*}$ \\
\hline High & $74(61.7)$ & $N R$ & 64.3 & & 48 & 34.8 & \\
\hline ITM2A & & & & & & & \\
\hline Low & $64(53.3)$ & $N R$ & 66.7 & $0.016^{*}$ & 48 & 31.3 & $<0.001^{*}$ \\
\hline High & $56(46.7)$ & NR & 92.9 & & NR & 92.9 & \\
\hline
\end{tabular}

Categorical variables were expressed as number (percentage), NR - denotes not reached yet, ${ }^{*}$ log-rank test, $p<0.05$ is significant

MFAP5, which is associated with induction of EMT, which denotes the inverse association between autophagy and EMT in TNBC.

Similar results were detected by Zhou et al. [9], who showed that the levels of ITM2A mRNA level were markedly down-regulated in tissues of breast cancer in comparison with levels in non-neoplastic tissues.

Moreover, the results of Zhou et al. [9] were in line with ours, confirming that expression of ITM2A inhibited growth of breast cancer cells. ITM2A expression was in- versely associated with advanced age, advanced stage, and basal like phenotype, so it might help in the prediction of chemotherapy response [19].

Autophagy dysfunction was found in many cancers, which might lead to progression or suppression of oncogenesis according to the type of the tumour [20]. Activation of autophagy was found in actively growing tumours in sites of nutrient deprivation and hypoxia that needed autophagy for tumour growth, such as cancer of the pancreas [21]. 
Table 4. Association between MFAP5, ITM2A expression, disease recurrence, distant metastases, and survival rates in included triple-negative breast cancer patients

\begin{tabular}{|c|c|c|c|c|c|c|c|c|c|c|}
\hline \multirow[t]{2}{*}{ Characteristics } & \multirow[t]{2}{*}{ All $(n=120)$} & \multicolumn{2}{|c|}{ Local recurrence } & \multirow[t]{2}{*}{$p$-value } & \multicolumn{2}{|c|}{ Distant metastasis } & \multirow[t]{2}{*}{$p$-value } & \multicolumn{2}{|c|}{ Survival } & \multirow[t]{2}{*}{$p$-value } \\
\hline & & $\begin{array}{c}\text { No } \\
(n=74)\end{array}$ & $\begin{array}{c}\text { Yes } \\
(n=14)\end{array}$ & & $\begin{array}{c}\text { No } \\
(n=88)\end{array}$ & $\begin{array}{c}\text { Yes } \\
(n=32)\end{array}$ & & $\begin{array}{l}\text { Alive } \\
(n=74)\end{array}$ & $\begin{array}{c}\text { Died } \\
(n=46)\end{array}$ & \\
\hline \multicolumn{11}{|l|}{ MFAP5 } \\
\hline Mean \pm SD & $46.58 \pm 32.90$ & $25.02 \pm 20.63$ & $66.85 \pm 13.19$ & \multirow[t]{2}{*}{$<0.001^{a}$} & $31.68 \pm 24.90$ & $87.56 \pm 6.21$ & \multirow{2}{*}{$<0.001^{a}$} & $26.10 \pm 22.90$ & $79.52 \pm 14.37$ & \multirow[t]{2}{*}{$<0.001^{a}$} \\
\hline Median (range) & $47.50(0-97)$ & $22(0-60)$ & $75(50-80)$ & & $24(0-80)$ & 89.5 (80-97) & & $22(0-80)$ & $80(50-97)$ & \\
\hline Low, n (\%) & $46(38.3)$ & $46(100)$ & $0(0)$ & \multirow[t]{2}{*}{$0.003^{b}$} & 46 (100) & $0(0)$ & \multirow[t]{2}{*}{$<0.001^{b}$} & $23(100)$ & $0(0)$ & \multirow[t]{2}{*}{$<0.001^{b}$} \\
\hline High, n (\%) & $74(61.7)$ & $28(66.7)$ & $14(33.3)$ & & $21(56.8)$ & $16(43.2)$ & & $14(37.8)$ & $46(62.2)$ & \\
\hline \multicolumn{11}{|l|}{ ITM2A } \\
\hline Mean $\pm S D$ & $40 \pm 33.94$ & $54.18 \pm 31.89$ & $40.57 \pm 20.49$ & \multirow[t]{2}{*}{$0.221^{a}$} & $52.02 \pm 31.73$ & $6.93 \pm 7.46$ & \multirow[t]{2}{*}{$<0.001^{\mathrm{a}}$} & $53.70 \pm 32.40$ & $17.95 \pm 23.41$ & \multirow[t]{2}{*}{$<0.001^{a}$} \\
\hline Median (range) & $25(0-90)$ & $70(0-90)$ & $23(22-90)$ & & $70(0-90)$ & $5(0-20)$ & & $70(0-90)$ & $12(0-90)$ & \\
\hline Low, n (\%) & 64 (53.3) & $22(68.8)$ & $10(31.3)$ & \multirow[t]{2}{*}{$0.035^{b}$} & $32(50)$ & $32(50)$ & \multirow[t]{2}{*}{$<0.001^{b}$} & $22(34.4)$ & $42(65.6)$ & \multirow[t]{2}{*}{$<0.001^{\text {b }}$} \\
\hline High, $n(\%)$ & $56(46.7)$ & $52(92.9)$ & $4(7.1)$ & & $56(100)$ & $0(0)$ & & $52(92.9)$ & $4(7.1)$ & \\
\hline
\end{tabular}

${ }^{a}$ Mann-Whitney $U$ test, ${ }^{b} \chi^{2}$ test or $\chi^{2}$ test for trend, $p<0.05$ is significant
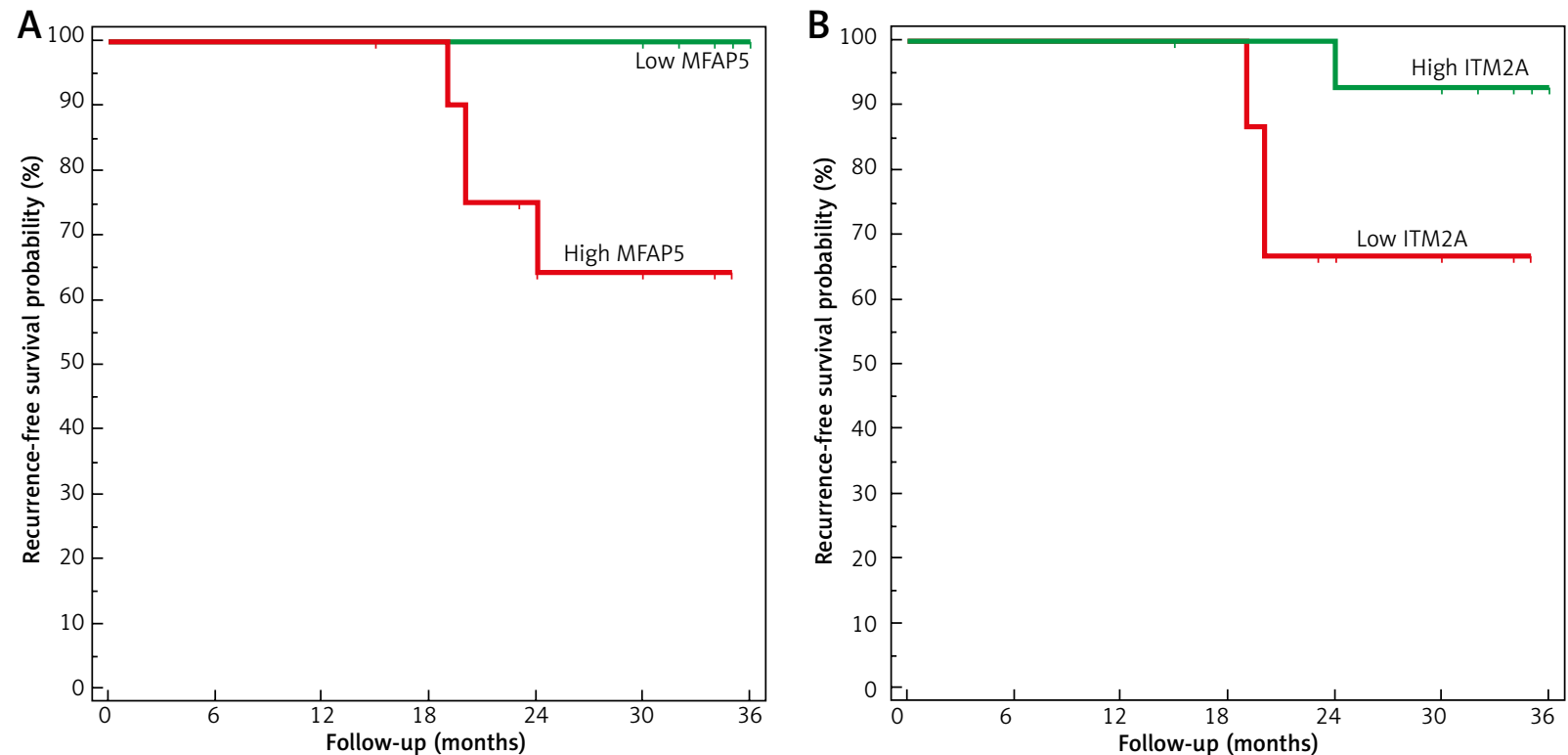

Fig. 4. Kaplan-Meier curve of local recurrence-free survival rate of the included triple-negative breast cancer patients: (A) stratified according to MFAP5 (B) stratified according to ITM2A

Hence, pharmacological or genetic autophagy inhibition leads to increased levels of reactive oxygen species and increased DNA damage, which in turn leads to the regulation of cell proliferation and survival [22].

In the current study we clarified the tumour suppressor function of autophagy and the association between up-regulation of ITM2A and prevention of TNBC progression, which was in line with [23, 24], and we associated its role in inhibition of EMT.

\section{Conclusions}

We assessed the expression of two novel biomarkers with different mechanisms of action, i.e. EMT induction and autophagy induction, in a specific molecular subtype of breast cancer (TNBC), and we concluded that EMT induction, which is denoted by MFAP5 up-regulation, was associated with poor clinicopathological parameters, disease progression, and unfavourable survival rates, while autophagy induction denoted by high ITM2A expression was associated with favourable clinicopathological parameters, lower incidence of disease progression, and favourable survival rates. Hence, we highlighted the possibility of the presence of a biological role of EMT and autophagy in controlling TNBC progression. Proving our results requires further studies to allow the discovery of novel, promising therapeutic targets for patients with breast cancer, particularly the TNBC molecular subtype, in the future.

\section{Strengths of the study}

Assessment of expression of novel markers in a large number of cases of breast cancer with a specific aggressive molecular subtype-TNBC. 

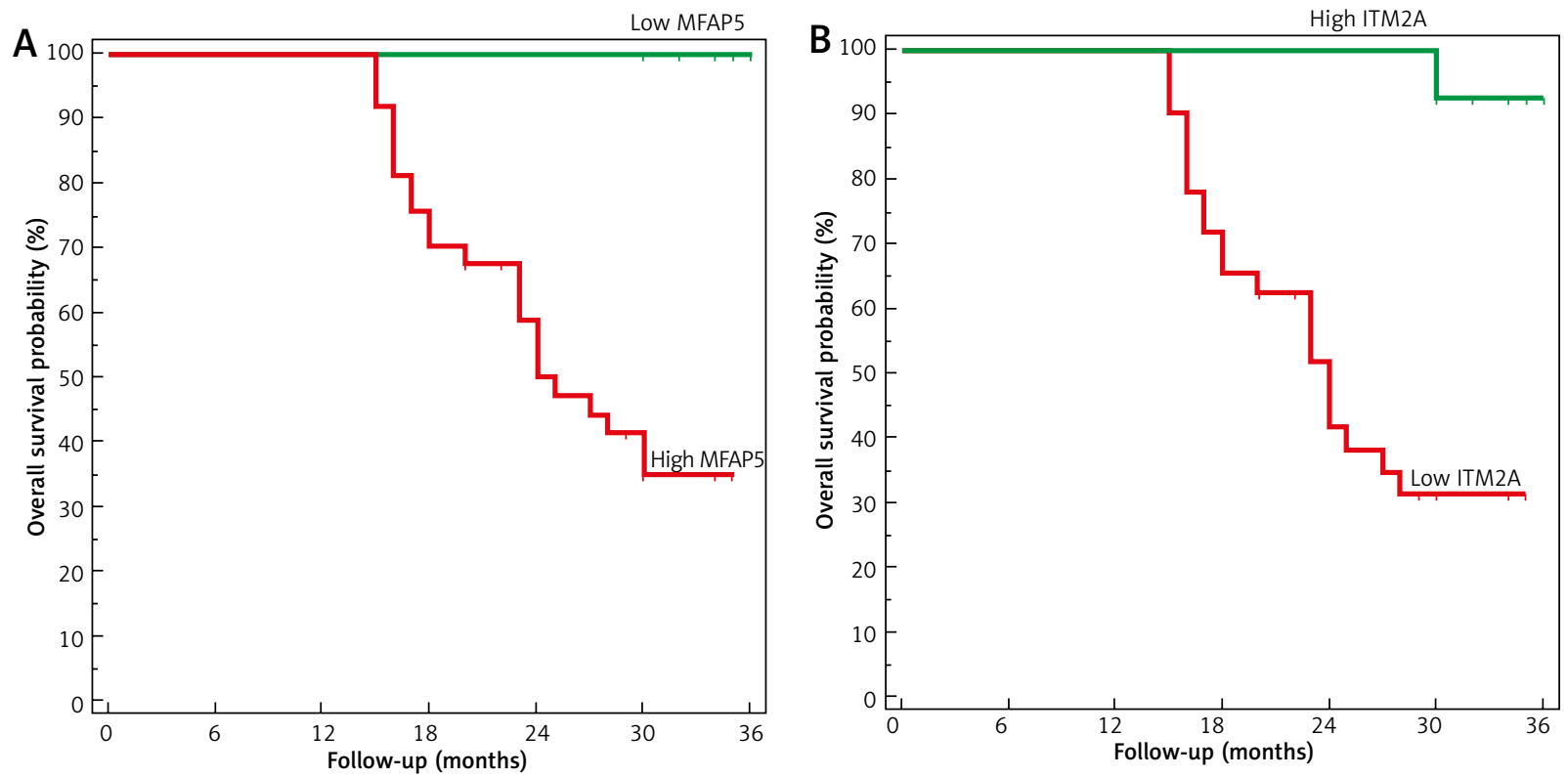

Fig. 5. Kaplan-Meier plot of overall survival rate of the included triple-negative breast cancer patients: (A) stratified according to MFAP5, (B) stratified according to ITM2A

\section{Limitations of the study}

Evaluation of the biomarkers using only a single meth od of assessment, i.e. immunohistochemistry, its retrospective nature, and the follow-up, i.e. only three years.

\section{Recommendations}

We recommend the assessment of MFAP5 and ITM2A expression in a large multicentre study includeing a large number of patients with a longer period of follow-up and using other methods of evaluation, such as genetic methods.

The authors declare no conflict of interest.

\section{References}

1. Wu Y, Wu P, Zhang Q, Chen W, Liu X, Zheng W. MFAP5 promotes basal-like breast cancer progression by activating the EMT program. Cell Biosci 2019; 9: 24.

2. Bianchini G, Balko J, Mayer I, Sanders ME, Gianni L. Triple-negative breast cancer: challenges and opportunities of a heterogeneous disease. Nat Rev Clin Oncol 2016; 13: 674.

3. Chung S, Jin Y, Han B, Qu Y, Gao B, Giuliano AE, Cui X. Identification of EGF-NF-KB-FOXC1 signaling axis in basal-like breast cancer. Cell Commun Signal 2017; 15: 22

4. Matysiak M, Kapka-Skrzypczak L, Jodlowska-Jedrych B, Kruszews ki M. EMT promoting transcription factors as prognostic markers in human breast cancer. Arch Gynecol Obstet 2017; 295: 817-825.

5. Lamouille S, Xu J, Derynck R. Molecular mechanisms of epithelialmesenchymal transition. Nat Rev Mol Cell Biol 2014; 15: 178-196.

6. Ceder R, Haig Y, Merne M, et al. Differentiation-promoting culture of competent and noncompetent keratinocytes identifies biomarkers for head and neck cancer. Am J Pathol 2012; 180: 457-472.
7. Yang X, Wu K, Li S, et al. MFAP5 and TNNC1: potential markers for predicting occult cervical lymphatic metastasis and prognosis in early stage tongue cancer. Oncotarget 2016; 8: 2525-2535.

8. Galluzzi L, Green DR. Autophagy-independent functions of the autophagy machinery. Cell 2019; 177: 1682-1699.

9. Zhou C, Wang M, Yang J, Xiong H, Wang Y, Tang J. Integral membrane protein $2 \mathrm{~A}$ inhibits cell growth in human breast cancer via enhancing autophagy induction. Cell Commun Signal 2019; 17: 105.

10. Edge SB, Compton CCS. The American Joint Committee on Cancer: the $7^{\text {th }}$ edition of the AJCC cancer staging manual and the future of TNM. Ann Surg Oncol 2010; 17: 1471-1474.

11. Elston CW, Ellis IO. Pathological prognostic factors in breast cancer. I. The value of histological grade in breast cancer: experience from a large study with long-term follow-up. Histopathology 1991; 19: 403-410

12. Puisieux A, Brabletz T, Caramel J. Oncogenic roles of EMT-inducing transcription factors. Nat Cell Biol 2014; 16: 488-494.

13. Bhosale PG, Cristea S, Ambatipudi S, et al. Chromosomal alterations and gene expression changes associated with the progression of leukoplakia to advanced gingivobuccal cancer. Transl Oncol 2017; 10: 396-409.

14. Yang X, Wu K, Li S, et al. MFAP5 and TNNC1: potential markers for predicting occult cervical lymphatic metastasis and prognosis in early stage tongue cancer. Oncotarget 2016; 8: 2525-2535.

15. Wu Y, Li X, Hu W, Wang N, Gao L, Chen Z, Zheng W. Microfibril-as sociated protein 5 (MFAP5): a potential novel marker in the extracellular matrix distinguishing basal-like breast carcinoma from breast cancer. Anal Quant Cytopathol 2016; 28: 175-182.

16. Leung CS, Yeung TL, Yip KP, et al. Calcium-dependent FAK/ CREB/ TNNC1 signalling mediates the effect of stromal MFAP5 on ovarian cancer metastatic potential. Nat Commun 2015; 5: 5092.

17. Marti P, Stein C, Blumer T, et al. YAP promotes proliferation, chemoresistance, and angiogenesis in human cholangiocarcinoma through TEAD transcription factors. Hepatology 2015; 62: 1497 1510

18. Nguyen TM, Shin IW, Lee TJ, Park J, Kim JH, Park MS, Lee EJ. Loss of ITM2A, a novel tumor suppressor of ovarian cancer through G2/M cell cycle arrest, is a poor prognostic factor of epithelial ovarian cancer. Gynecol Oncol 2016; 140: 545-553. 
19. Parise CA, Caggiano V. Breast Cancer survival defined by the ER/ PR/HER2 subtypes and a surrogate classification according to tumor grade and Immunohistochemical biomarkers. J Cancer Epidemiol 2014; 2014: 469251.

20. Kimmelman AC. The dynamic nature of autophagy in cancer. Genes Dev 2011; 25: 1999-2010.

21. Yang S, Wang X, Contino G, et al. Pancreatic cancers require autophagy for tumor growth. Genes Dev 2011; 25: 717-729.

22. Kimmelman AC, White E. Autophagy and tumor metabolism. Cell Metab 2017; 25: 1037-1043.

23. Yu L, McPhee CK, Zheng L, et al. Termination of autophagy and reformation of lysosomes regulated by mTOR. Nature 2010; 465: 942-946.

24. Kim J, Kundu M, Viollet B, Guan KL. AMPK and mTOR regulate autophagy through direct phosphorylation of Ulk1. Nat Cell Biol 2011; 13: 132-141.

\section{Address for correspondence}

\section{Ola A. Harb}

Department of Pathology

Faculty of Medicine

Zagazig University

11111 Zagazig, Egypt

e-mail: olaharb2015@gmail.com

Submitted: 16.05 .2020

Accepted: 19.06 .2020 